\title{
GEOMETRIC PROBABILITIES CONCERNING LARGE RANDOM TRIANGLES IN THE HYPERBOLIC PLANE
}

\author{
YUKINAO ISOKAWA
}

\begin{abstract}
Concerning a random triangle on a disk $D_{R}$ of radius $R$ in the hyperbolic plane, the following four geometric probabilities are studied: (i) the probability $p_{a}(R)$ that a random triangle is acute; (ii) the probability $p_{o}(R)$ that a random triangle has the orthocenter; (iii) the probability $p_{e}(R)$ that a random triangle has at least one of the three excenters; and (iv) the probability $p_{c}(R)$ that a random triangle has the crrcumcenter. It is shown that, as $R$ tends to the infinity, both the probability $p_{a}(R)$ and $p_{o}(R)$ tend to one, whereas the probability $p_{e}(R)$ tends to zero. Moreover it is shown that the probability $p_{c}(R)$ tends to a limit $p_{c}$, which can be expressed as a certain expectation concerning a random triangle in the Euclidean plane. To evaluate this expectation numerically, we obtain 0.45962039 as an estimate for $p_{c}$.
\end{abstract}

\section{Introduction}

The first problem concerning random triangles in the Euclidean plane is perhaps the problem "what is the probability that a random triangle is acute?". This problem was proposed by [11], and as an answer to the problem, it gave the probability $4 / \pi^{2}-1 / 8$, assuming that three vertices of a random triangle are distributed independently and uniformly in the unit disk. Since that time various studies have been made on this problem. In [5] five different solutions to the problem are given. Whereas differences of these solutions reflect those of meanings on random triangles, they lead to the common probability $1 / 4$. In [10] one more solution to the problem is given, where three vertices are assumed to be distributed according as a Gaussian distribution. The answer is again 1/4. These results are extended in [6] and [3] to the corresponding problem for random triangles in higher-dimensional Euclidean spaces.

Another problem oncerning random triangles, which has been extensively studied, is to find the distribution of the area of a random triangle whose three vertices are uniformly distributed in a given domain of Euclidean spaces. In [1], for the case that a domain is a triangle in the Euclidean plane, explicit expressions for all the moments of the area are given. In [2] these results are

Received October 10, 1998; revised July 22, 1999. 
partially extended to the case that a domain is a simplex in higher-dimensional Euclidean spaces.

Moreover, in the field of random triangles in Euclidean spaces, many other directions of investigations have been successfully performed (for example, see [7], [8], and [9]). On the other hand, as long as the present author searches existing literatures, there seem to be little research on random triangles in the hyperbolic plane.

In the hyperbolic plane, triangles have somewhat mysterious properties which those in the Euclidean plane do not have (see [4]). Whereas they always have centroids and incenters, they do not always have circumcenters, orthocenters and excenters. Hence a problem arises: with what probabilities does a random triangle in the hyperbolic plane has these "geometric" centers? This is the problem which we investigate in this paper.

In Section 2 we consider a random triangle $\mathrm{ABC}$ on a disk of radius $R$ and centered at the origin of the hyperbolic plane, and study probability distribution of lengths of three sides of the random triangle. We prove that this probability distribution converges to a limit distribution as $R$ tends to the infinity, and that the limit distribution has a probability density which can be expressed as a double integral. In Section 3, we consider the following geometric probabilities:

1. The probability $p_{a}(R)$ that a random triangle ABC is acute,

2. The probability $p_{o}(R)$ that a random triangle $\mathrm{ABC}$ has the orthocenter,

3. The probability $p_{e}(R)$ that a random triangle $\mathrm{ABC}$ has at least one of the three excenters,

4. The probability $p_{c}(R)$ that a random triangle $\mathrm{ABC}$ has the circumcenter.

About these geometric probabilities, we first show that, as $R$ tends to the infinity, both $p_{a}(R)$ and $p_{o}(R)$ tend to one, $p_{e}(R)$ tends to zero, whereas $p_{c}(R)$ converges to a limit $p_{c}$ such that $0<p_{c}<1$. Moreover we prove that the limit probability $p_{c}$ can be expressed as a certain expectation concerning a random triangle in the Euclidean plane. In Section 4 we evaluate this expectation numerically. Our estimate for $p_{c}$ is about 0.45962039 . Roughly speaking, we may say that the majority of large random triangles in the hyperbolic plane are acute, have the orthocenters, but have no excenters, and that about $46 \%$ of large random triangles have the circumcenters.

\section{Limit probability distribution of lengths of three sides of a random triangle}

We begin by clarifying a concept of a random point. Let $\boldsymbol{H}^{2}$ be a hyperbolic plane whose metric $d s$ is given by polar coordinate as $d s^{2}=d r^{2}+$ $\sinh ^{2} r d \theta$, and $D_{R}$ a disk of radius $R$ centered at the origin $\mathrm{O}$ of $H^{2}$. We say that $\mathrm{P}$ is a random point on the disk $D_{R}$ when $\mathrm{P}$ is uniformly distributed on the disk. To state precisely, let us denote a polar coordinate of $\mathrm{P}$ by $\left(r_{\mathbf{P}}, \theta_{\mathrm{P}}\right)$. Then we say that $\mathrm{P}$ is a random point on the disk $D_{R}$ when $r_{\mathrm{P}}$ is distributed 
according to a probability density $\sinh r d r / L$ on an interval $(0, R)$, where $L=$ $\cosh R-1$, and $\theta_{\mathrm{P}}$ is uniformly distributed on an interval $[0,2 \pi)$.

Now we consider a triangle $\mathrm{ABC}$ whose three vertices $\mathrm{A}, \mathrm{B}$, and $\mathrm{C}$ are mutually independent random points on the disk $D_{R}$. To state explicitly, denoting polar coordinates of three points $\mathrm{A}, \mathrm{B}$, and $\mathrm{C}$ by $\left(r_{\mathrm{A}}, \theta_{\mathrm{A}}\right),\left(r_{\mathrm{B}}, \theta_{\mathrm{B}}\right)$, and $\left(r_{\mathrm{C}}, \theta_{\mathrm{C}}\right)$ respectively, we assume that

1. $r_{\mathrm{A}}, \theta_{\mathrm{A}}, r_{\mathrm{B}}, \theta_{\mathrm{B}}, r_{\mathrm{C}}, \theta_{\mathrm{C}}$ are mutually independent.

2. $r_{\mathrm{A}}, r_{\mathrm{B}}, r_{\mathrm{C}}$ have a common probability density $\sinh r d r / L$.

3. $\theta_{\mathrm{A}}, \theta_{\mathrm{B}}, \theta_{\mathrm{C}}$ have the uniform distribution on an interval $[0,2 \pi)$.

In this section we investigate a probability distribution of lengths of three sides of a "large" random triangle. To state precisely, for a random triangle ABC on the disk $D_{R}$, let us denote lengths of its three sides by $a, b, c$, and introduce

$$
X_{L}=\frac{\cosh a}{L^{2}}, \quad Y_{L}=\frac{\cosh b}{L^{2}}, \quad Z_{L}=\frac{\cosh c}{L^{2}} .
$$

We study the limit probability distribution of $\left(X_{L}, Y_{L}, Z_{L}\right)$ when $L$ tends to the infinity. For this purpose, we define the characteristic function of $\left(X_{L}, Y_{L}, Z_{L}\right)$,

$$
f_{L}\left(t_{1}, t_{2}, t_{3}\right)=\mathrm{E}\left[\exp \left(i\left(t_{1} X_{L}+t_{2} Y_{L}+t_{3} Z_{L}\right)\right)\right] .
$$

Then we obtain the following lemma.

LEMMA 1. The limit characteristic function

$$
f\left(t_{1}, t_{2}, t_{3}\right)=\lim _{L \rightarrow \infty} f_{L}\left(t_{1}, t_{2}, t_{3}\right)
$$

exists and it can be expressed as

$$
\begin{aligned}
f\left(t_{1}, t_{2}, t_{3}\right)= & \frac{1}{4 \pi^{2}} \int_{0}^{2 \pi} \int_{0}^{2 \pi} d \phi d \psi \int_{0}^{1} \int_{0}^{1} \int_{0}^{1} d u d v d w \\
& \cdot \exp \left[i t_{1}(1-\lambda) v w+i t_{2}(1-\mu) w u+i t_{3}(1-v) u v\right] .
\end{aligned}
$$

Proof. Without loss of generality, we may assume that the vertex A lies on the $x$-axis. We denote the angles which the line segments OB and OC make with the $x$-axis by $\phi$ and $\psi$ respectively. Then, by hyperbolic trigonometry, we have

$$
\left\{\begin{array}{l}
L^{2} \cdot X_{L}=\cosh a=\cosh r_{\mathrm{B}} \cosh r_{\mathrm{C}}-\sinh r_{\mathrm{B}} \sinh r_{\mathrm{C}} \cos (\psi-\phi) \\
L^{2} \cdot Y_{L}=\cosh b=\cosh r_{\mathrm{C}} \cosh r_{\mathrm{A}}-\sinh r_{\mathrm{C}} \sinh r_{\mathrm{A}} \cos \psi \\
L^{2} \cdot Z_{L}=\cosh c=\cosh r_{\mathrm{A}} \cosh r_{\mathrm{B}}-\sinh r_{\mathrm{A}} \sinh r_{\mathrm{B}} \cos \phi .
\end{array}\right.
$$

At this point it is convenient to introduce the following variables:

$$
\lambda=\cos (\psi-\phi), \mu=\cos \psi, v=\cos \phi
$$

and

$$
u=\cosh r_{\mathrm{A}} / L, v=\cosh r_{\mathrm{B}} / L, w=\cosh r_{\mathrm{C}} / L
$$


Then $X_{L}, Y_{L}, Z_{L}$ can be written as

$$
\left\{\begin{array}{l}
X_{L}=v w-\lambda \sqrt{v^{2}-\frac{1}{L^{2}}} \sqrt{w^{2}-\frac{1}{L^{2}}} \\
Y_{L}=w u-\mu \sqrt{w^{2}-\frac{1}{L^{2}}} \sqrt{u^{2}-\frac{1}{L^{2}}} \\
Z_{L}=u v-v \sqrt{u^{2}-\frac{1}{L^{2}}} \sqrt{v^{2}-\frac{1}{L^{2}}}
\end{array}\right.
$$

Moreover, it is easy to see that all $u, v$, and $w$ have simply the uniform distribution on an interval $(1 / L, 1+1 / L)$. Consequently, noting that both $\phi$ and $\psi$ have the uniform distribution on an interval $(0,2 \pi)$, we have

$$
\begin{aligned}
f_{L}\left(t_{1}, t_{2}, t_{3}\right)= & \frac{1}{4 \pi^{2}} \int_{0}^{2 \pi} \int_{0}^{2 \pi} d \phi d \psi \int_{1 / L}^{1+1 / L} \int_{1 / L}^{1+1 / L} \int_{1 / L}^{1+1 / L} d u d v d w \\
& \cdot \exp \left[i t_{1}\left(v w-\lambda \sqrt{v^{2}-\frac{1}{L^{2}}} \sqrt{w^{2}-\frac{1}{L^{2}}}\right)\right. \\
& +i t_{2}\left(w u-\mu \sqrt{w^{2}-\frac{1}{L^{2}}} \sqrt{u^{2}-\frac{1}{L^{2}}}\right) \\
& \left.+i t_{3}\left(u v-v \sqrt{u^{2}-\frac{1}{L^{2}}} \sqrt{v^{2}-\frac{1}{L^{2}}}\right)\right]
\end{aligned}
$$

Then, using the bounded convergence theorem, we obtain the lemma.

Let $p(x, y, z)$ denote the probability density corresponding to the characteristic function $f\left(t_{1}, t_{2}, t_{3}\right)$. From Lemma 1 we can derive the following expression for $p(x, y, z)$.

\section{LEMMA 2.}

$$
p(x, y, z)=\frac{1}{8 \pi^{2} \sqrt{x y z}} \int_{0}^{2 \pi} \int_{0}^{2 \pi} I_{C}(\phi, \psi) \frac{d \phi d \psi}{\sqrt{(1-\lambda)(1-\mu)(1-v)}},
$$

where $\lambda, \mu, v$ are defined by (2.3) and $I_{C}$ denotes the indicator function of the set

$$
C=\left\{(\phi, \psi): \frac{y z}{x}<\frac{(1-\mu)(1-v)}{1-\lambda}, \frac{z x}{y}<\frac{(1-v)(1-\lambda)}{1-\mu}, \frac{x y}{z}<\frac{(1-\lambda)(1-\mu)}{1-v}\right\} .
$$

Proof. Consider a characteristic function corresponding to the probability density (2.4): 


$$
\tilde{f}\left(t_{1}, t_{2}, t_{3}\right)=\int_{0}^{\infty} \int_{0}^{\infty} \int_{0}^{\infty} p(x, y, z) e^{i\left(t_{1} x+t_{2} y+t_{3} z\right)} d x d y d z
$$

Note that if $(\phi, \psi) \in C$, we can derive $x<1-\lambda, y<1-\mu, z<1-v$. In particular we have $x<2, y<2, z<2$, which assures existence of $\tilde{f}$.

Now we change variables

$$
x=(1-\lambda) u v, \quad y=(1-\mu) v w, \quad z=(1-v) w u .
$$

Then we can see that the Jacobian

$$
\frac{\partial(x, y, z)}{\partial(u, v, w)}=2(1-\lambda)(1-\mu)(1-v) u v w
$$

and that the condition $(\phi, \psi) \in C$ is equivalent to the condition

$$
u<1, \quad v<1, \quad w<1 \text {. }
$$

Accordingly we obtain

$$
\begin{aligned}
\tilde{f}\left(t_{1}, t_{2}, t_{3}\right)= & \frac{1}{4 \pi^{2}} \int_{0}^{2 \pi} \int_{0}^{2 \pi} d \phi d \psi \int_{0}^{1} \int_{0}^{1} \int_{0}^{1} d u d v d w \\
& \cdot \exp \left[i t_{1}(1-\lambda) v w+i t_{2}(1-\mu) w u+i t_{3}(1-v) u v\right] .
\end{aligned}
$$

Thus $\tilde{f}$ is identical to $f$ and the proof is completed.

From Lemma 1 and Lemma 2 follows the following theorem.

THEOREM 1. Appropriately normalized lengths of three sides of a random triangle, $\left(X_{L}, Y_{L}, Z_{L}\right)$, have the limit probability distribution as the radius $R$ of the disk $D_{R}$, or equivalently $L=\cosh R-1$, tends to the infinity. Furthermore its probability density is given by (2.4).

\section{Geometric probabilities concerning a large random triangle}

In this section we study geometric probabilities $p_{a}(R), p_{o}(R), p_{e}(R)$ and $p_{c}(R)$ when $R$ tends to the infinity. Let us denote three sides and three angles of a triangle $\mathrm{ABC}$ by

$$
\begin{aligned}
& a=\mathrm{BC}, \quad b=\mathrm{CA}, \quad c=\mathrm{AB}, \\
& \alpha=\angle \mathrm{CAB}, \quad \beta=\angle \mathrm{ABC}, \quad \gamma=\angle \mathrm{BCA},
\end{aligned}
$$

and we put $x=\cosh a, y=\cosh b, z=\cosh c$.

Lemma 3. (i) $A$ triangle $\mathrm{ABC}$ is acute if and only if

$$
y z>x, \quad z x>y, \quad x y>z .
$$

(ii) A triangle $\mathrm{ABC}$ has the orthocenter if and only if

$$
3 x^{2} y^{2} z^{2}-2 x^{3} y z-2 x y^{3} z-2 x y z^{3}+y^{2} z^{2}+z^{2} x^{2}+x^{2} y^{2}>0 .
$$


(iii) A triangle $\mathrm{ABC}$ has the excenter beyond the side $\mathrm{BC}$ if and only if

$$
\begin{aligned}
& 2 x \sqrt{y^{2}-1} \sqrt{z^{2}-1}-2 y \sqrt{z^{2}-1} \sqrt{x^{2}-1} \\
& \quad-2 z \sqrt{x^{2}-1} \sqrt{y^{2}-1}+x^{2}+y^{2}+z^{2}-3<0 .
\end{aligned}
$$

(iv) $A$ triangle $\mathrm{ABC}$ has the circumcenter if and only if

$$
x^{2}+y^{2}+z^{2}-2 y z-2 z x-2 x y+2 x+2 y+2 z-3>0 .
$$

Proof. The assertion (i) is an easy consequence from the cosine formula of hyperbolic trigonometry. Now we prove (ii). To use the notations used in [4], as is shown in p. 121 and p. 131 of Chapter 6 of [4], a triangle $\mathrm{ABC}$ has the orthocenter if and only if

$$
(2 \cos \alpha \cos \beta \cos \gamma+1) a m_{s}^{2}<\sin ^{2} \alpha \sin ^{2} \beta \sin ^{2} \gamma,
$$

where

$$
\begin{aligned}
a m_{s}^{2}= & -16 \sin ^{2} \frac{\alpha}{2} \sin ^{2} \frac{\beta}{2} \cos ^{2} \frac{\gamma}{2} \\
+ & 4\left(\sin \frac{\alpha}{2}+\sin \frac{\beta}{2}-\cos \frac{\gamma}{2}\right)\left(-\sin \frac{\alpha}{2}+\sin \frac{\beta}{2}+\cos \frac{\gamma}{2}\right) \\
& \cdot\left(\sin \frac{\alpha}{2}-\sin \frac{\beta}{2}+\cos \frac{\gamma}{2}\right)\left(\sin \frac{\alpha}{2}+\sin \frac{\beta}{2}+\cos \frac{\gamma}{2}\right) .
\end{aligned}
$$

Then, by a lengthy but elementary calculation, we can show that the right hand side subtracted by the left hand side in the above inequality is equivalent to

$$
\begin{aligned}
& \left(x^{2}+y^{2}+z^{2}-2 x y z-1\right)^{2} \\
& \quad \cdot\left(3 x^{2} y^{2} z^{2}-2 x^{3} y z-2 x y^{3} z-2 x y z^{3}+y^{2} z^{2}+z^{2} x^{2}+x^{2} y^{2}\right) \\
& \quad /\left(\left(x^{2}-1\right)^{2}\left(y^{2}-1\right)^{2}\left(z^{2}-1\right)^{2}\right)>0 .
\end{aligned}
$$

Thus the assertion (ii) is proved.

Next we prove (iii). As is shown in p. 121 of [4], a triangle $A B C$ has the excenter beyond the side $\mathrm{BC}$ if and only if

$$
\sin \frac{\beta}{2}+\sin \frac{\gamma}{2}>\cos \frac{\alpha}{2}
$$

Then, by a similar calculation to that in the proof of (ii), we can show that the above inequality is equivalent to

$$
\begin{aligned}
& \left(2 x y z+1-x^{2}-y^{2}-z^{2}\right) \\
& \cdot\left(2 x \sqrt{y^{2}-1} \sqrt{z^{2}-1}-2 y \sqrt{z^{2}-1} \sqrt{x^{2}-1}\right. \\
& \left.\quad-2 z \sqrt{x^{2}-1} \sqrt{y^{2}-1}+x^{2}+y^{2}+z^{2}-3\right) \\
& /\left(\left(x^{2}-1\right)\left(y^{2}-1\right)\left(z^{2}-1\right)\right)>0 .
\end{aligned}
$$


Now, noting that $|b-c|<a<b+c$, we can easily see that

$$
2 x y z+1-x^{2}-y^{2}-z^{2}>0 .
$$

Accordingly (iii) is obtained.

Finally we prove (iv). As is stated in p. 118 of [4], a triangle $A B C$ has the circumcenter if and only if the following three inequalities hold:

$$
\sinh \frac{a}{2}<\sinh \frac{b}{2}+\sinh \frac{c}{2}, \sinh \frac{b}{2}<\sinh \frac{c}{2}+\sinh \frac{a}{2}, \sinh \frac{c}{2}<\sinh \frac{a}{2}+\sinh \frac{b}{2} .
$$

Obviously it is impossible that more than one of the above inequalities hold simultaneously. Now, by doing an elementary calculation again, we get

$$
\begin{aligned}
\left(-\sinh \frac{a}{2}+\sinh \frac{b}{2}+\sinh \frac{c}{2}\right)\left(\sinh \frac{a}{2}-\sinh \frac{b}{2}+\sinh \frac{c}{2}\right) \\
\cdot\left(\sinh \frac{a}{2}+\sinh \frac{b}{2}-\sinh \frac{c}{2}\right)\left(\sinh \frac{a}{2}+\sinh \frac{b}{2}+\sinh \frac{c}{2}\right) \\
=-\frac{1}{4}\left(x^{2}+y^{2}+z^{2}-2 y z-2 z x-2 x y+2 x+2 y+2 z-3\right) .
\end{aligned}
$$

Hence immediately follows (iv).

Using the above Lemma 3 and Theorem 1, we obtain the following theorem.

THEOREM 2.

$$
\begin{aligned}
& \lim _{R \rightarrow \infty} p_{a}(R)=1 . \\
& \lim _{R \rightarrow \infty} p_{o}(R)=1 . \\
& \lim _{R \rightarrow \infty} p_{e}(R)=0 .
\end{aligned}
$$

Proof. Since proofs of (i) and (ii) can be carried out in similar ways to that of (iii), we will prove only (iii). Let $p_{e, 1}(R)$ stand for the probability that a random triangle $\mathrm{ABC}$ has the excenter beyond the side $\mathrm{BC}$. In order to prove (iii), it suffices to prove that

$$
\lim _{R \rightarrow \infty} p_{e, 1}(R)=0
$$

because $p_{e}(R) \leq 3 p_{e, 1}(R)$.

Now, recalling (2.2), we replace $x, y, z$ in (iii) of Lemma 3 by $L^{2} X_{L}, L^{2} Y_{L}, L^{2} Z_{L}$ respectively. Then we have

$$
p_{e, 1}(R)=P\left\{\left(X_{L}, Y_{L}, Z_{L}\right) \in A_{\varepsilon}\right\},
$$

where $\varepsilon$ denotes for $1 / L^{2}$ and 
Since

$$
\begin{gathered}
A_{\varepsilon}=\left\{(x, y, z) \in \boldsymbol{R}^{3}: x \sqrt{y^{2}-\varepsilon^{2}} \sqrt{z^{2}-\varepsilon^{2}}-y \sqrt{z^{2}-\varepsilon^{2}} \sqrt{x^{2}-\varepsilon^{2}}\right. \\
\left.-z \sqrt{x^{2}-\varepsilon^{2}} \sqrt{y^{2}-\varepsilon^{2}}+\frac{\varepsilon}{2}\left(x^{2}+y^{2}+z^{2}\right)-\frac{3}{2} \varepsilon^{3}>0\right\} .
\end{gathered}
$$

$$
\begin{aligned}
& x \sqrt{y^{2}-\varepsilon^{2}} \sqrt{z^{2}-\varepsilon^{2}}-y \sqrt{z^{2}-\varepsilon^{2}} \sqrt{x^{2}-\varepsilon^{2}}-z \sqrt{x^{2}-\varepsilon^{2}} \sqrt{y^{2}-\varepsilon^{2}} \\
& \quad<x y z-y(z-\varepsilon)(x-\varepsilon)-z(x-\varepsilon)(y-\varepsilon) \\
& \quad<-x y z+\varepsilon(x y+2 y z+z x),
\end{aligned}
$$

we have $A_{\varepsilon} \subset \bar{A}_{\varepsilon}$, where

$$
\bar{A}_{\varepsilon}=\left\{(x, y, z) \in \boldsymbol{R}^{3}:-x y z+\frac{\varepsilon}{2}\left(x^{2}+y^{2}+z^{2}+2 x y+4 y z+2 z x\right)>0\right\} .
$$

Hence

$$
p_{e, 1}(R) \leq P\left\{\left(X_{L}, Y_{L}, Z_{L}\right) \in \bar{A}_{\varepsilon}\right\} .
$$

Now let us take an arbitary positive number $\varepsilon_{0}$. Then, noting that $\bar{A}_{\varepsilon} \subset \bar{A}_{\varepsilon_{0}}$ for any $\varepsilon$ such that $\varepsilon<\varepsilon_{0}$, and using Theorem 1, we get

$$
\begin{aligned}
\varlimsup_{R \rightarrow \infty} P\left\{\left(X_{L}, Y_{L}, Z_{L}\right) \in \bar{A}_{\varepsilon}\right\} & \leq \lim _{R \rightarrow \infty} P\left\{\left(X_{L}, Y_{L}, Z_{L}\right) \in \bar{A}_{\varepsilon_{0}}\right\} \\
& =\iiint_{\bar{A}_{\varepsilon_{0}}} p(x, y, z) d x d y d z .
\end{aligned}
$$

Letting $\varepsilon_{0}$ tend to zero in the above integral, we can see that this integral will vanish. Therefore the assertion (iii) is confirmed.

As for the probability $p_{c}(R)$, we have the following Lemma 4 . To state the lemma, we introduce a quadratic form

$$
Q(x, y, z)=x^{2}+y^{2}+z^{2}-2 y z-2 z x-2 x y,
$$

and an integral

$$
I(a, b, c)=\iiint_{\{(x, y, z): Q(x, y, z)>0, x>a, y>b, z>c\}} \frac{d x d y d z}{x^{2} y^{2} z^{2}} .
$$

Furthermore, assuming existence of $\lim _{R \rightarrow \infty} p_{c}(R)$, we denote it by $p_{c}$.

LEMMA 4. The limit probability $p_{c}$ exists and

$$
\begin{aligned}
1-p_{c}= & \frac{1}{4 \pi^{2}} \int_{0}^{2 \pi} \int_{0}^{2 \pi} \frac{d \phi d \psi}{\sqrt{(1-\lambda)(1-\mu)(1-v)}} \\
& \cdot I\left(\sqrt{\frac{1-\lambda}{(1-\mu)(1-v)}}, \sqrt{\frac{1-\mu}{(1-v)(1-\lambda)}}, \sqrt{\frac{1-v}{(1-\lambda)(1-\mu)}}\right) .
\end{aligned}
$$


Proof. Replacing $x, y, z$ in (iv) of Lemma 3 by $L^{2} X_{L}, L^{2} Y_{L}, L^{2} Z_{L}$ respectively, we have

$$
p_{c}(R)=P\left\{\left(X_{L}, Y_{L}, Z_{L}\right) \in A_{\varepsilon}\right\},
$$

where $\varepsilon$ denotes for $1 / L^{2}$ and

$$
A_{\varepsilon}=\left\{(x, y, z) \in \boldsymbol{R}^{3}: Q(x, y, z)+2 \varepsilon(x+y+z)-3 \varepsilon^{2}<0\right\} .
$$

Now we introduce

$$
\begin{aligned}
& \bar{A}_{\varepsilon}=\left\{(x, y, z) \in \boldsymbol{R}^{3}: Q(x, y, z)-3 \varepsilon^{2}<0\right\} \\
& \underline{A}_{\varepsilon}=\left\{(x, y, z) \in \boldsymbol{R}^{3}: Q(x, y, z)+2 \varepsilon(x+y+z)<0\right\} .
\end{aligned}
$$

Then, for any $\varepsilon$ such that $\varepsilon<\varepsilon_{0}$, we have

$$
\underline{A}_{\varepsilon_{0}} \subset \underline{A}_{\varepsilon} \subset A_{\varepsilon} \subset \bar{A}_{\varepsilon} \subset \bar{A}_{\varepsilon_{0}} .
$$

Consequently Theorem 1 implies that

$$
\begin{aligned}
& \iiint_{\underline{A}_{\varepsilon_{0}}} p(x, y, z) d x d y d z=\lim _{R \rightarrow \infty} \boldsymbol{P}\left\{\left(X_{L}, Y_{L}, Z_{L}\right) \in \underline{A}_{\varepsilon_{0}}\right\} \\
& \quad \leq \varliminf_{R \rightarrow \infty} \boldsymbol{P}\left\{\left(X_{L}, Y_{L}, Z_{L}\right) \in A_{\varepsilon}\right\} \\
& \quad \leq \varlimsup_{R \rightarrow \infty} \boldsymbol{P}\left\{\left(X_{L}, Y_{L}, Z_{L}\right) \in A_{\varepsilon}\right\} \\
& \quad \leq \lim _{R \rightarrow \infty} \boldsymbol{P}\left\{\left(X_{L}, Y_{L}, Z_{L}\right) \in \bar{A}_{\varepsilon_{0}}\right\}=\iiint_{\bar{A}_{\varepsilon_{0}}} p(x, y, z) d x d y d z
\end{aligned}
$$

Hence follows the existence of the limit $p_{c}$ and

$$
p_{c}=\iiint_{\{(x, y, z): Q(x, y, z)<0\}} p(x, y, z) d x d y d z .
$$

Thus, using Lemma 2, we have

$$
\begin{gathered}
1-p_{c}=\frac{1}{8 \pi^{2}} \iiint_{\{Q(x, y, z) \geq 0\}} \frac{d x d y d z}{\sqrt{x y z}} \\
\int_{0}^{2 \pi} \int_{0}^{2 \pi} I_{C}(\phi, \psi) \frac{d \phi d \psi}{\sqrt{(1-\lambda)(1-\mu)(1-v)}} .
\end{gathered}
$$

Now we change variables

$$
x=\frac{1}{\eta \zeta}, y=\frac{1}{\zeta \xi}, z=\frac{1}{\xi \eta} .
$$

Then, since

$$
\frac{\partial(x, y, z)}{\partial(\xi, \eta, \zeta)}=-\frac{2}{\xi^{3} \eta^{3} \zeta^{3}}
$$


and

$$
Q(x, y, z)=\frac{1}{\xi^{2} \eta^{2} \zeta^{2}} Q(\xi, \eta, \zeta)
$$

we obtain (3.3). Thus the proof is completed.

The triple integral (3.2) can be evaluate as follows.

Lemma 5. Assume that

$$
\sqrt{a}<\sqrt{b}+\sqrt{c}, \quad \sqrt{b}<\sqrt{c}+\sqrt{a}, \quad \sqrt{c}<\sqrt{a}+\sqrt{b} .
$$

Then

$$
\begin{aligned}
I(a, b, c)= & \left(\frac{1}{a^{2} b}+\frac{1}{a b^{2}}+\frac{1}{b^{2} c}+\frac{1}{b c^{2}}+\frac{1}{c^{2} a}+\frac{1}{c a^{2}}\right) \\
& -2\left(\frac{1}{a^{5 / 2} \sqrt{b}}+\frac{1}{\sqrt{a} b^{5 / 2}}+\frac{1}{b^{5 / 2} \sqrt{c}}+\frac{1}{\sqrt{b} c^{5 / 2}}+\frac{1}{c^{5 / 2} \sqrt{a}}+\frac{1}{\sqrt{c} a^{5 / 2}}\right) \\
& -\frac{2}{3}\left(\frac{1}{a^{3 / 2} b^{3 / 2}}+\frac{1}{b^{3 / 2} c^{3 / 2}}+\frac{1}{c^{3 / 2} a^{3 / 2}}\right) \\
& +2\left(\frac{1}{a^{3}}+\frac{1}{b^{3}}\right) \log \left(\frac{\sqrt{a}+\sqrt{b}}{\sqrt{c}}\right)+2\left(\frac{1}{b^{3}}+\frac{1}{c^{3}}\right) \log \left(\frac{\sqrt{b}+\sqrt{c}}{\sqrt{a}}\right) \\
& +2\left(\frac{1}{c^{3}}+\frac{1}{a^{3}}\right) \log \left(\frac{\sqrt{c}+\sqrt{a}}{\sqrt{b}}\right) .
\end{aligned}
$$

Proof. Without loss of generality we may assume that $a \geq b \geq c$. We first consider an integral

$$
K(x, y)=\int_{\{z: Q(x, y, z)>0, z>c\}} \frac{d z}{z^{2}} .
$$

It is easy to see that $Q(x, y, z)>0$ if and only if

$$
z>(\sqrt{x}+\sqrt{y})^{2} \text { or } z<(\sqrt{x}-\sqrt{y})^{2} .
$$

Thus, if $(\sqrt{x}-\sqrt{y})^{2}>c$,

$$
\begin{aligned}
K(x, y) & =\left(\int_{(\sqrt{x}+\sqrt{y})^{2}}^{\infty}+\int_{c}^{(\sqrt{x}-\sqrt{y})^{2}}\right) \frac{d z}{z^{2}} \\
& =\frac{1}{(\sqrt{x}+\sqrt{y})^{2}}+\frac{1}{c}-\frac{1}{(\sqrt{x}-\sqrt{y})^{2}} .
\end{aligned}
$$

On the other hand, if $(\sqrt{x}-\sqrt{y})^{2}<c$, 


$$
K(x, y)=\int_{(\sqrt{x}+\sqrt{y})^{2}}^{\infty} \frac{d z}{z^{2}}=\frac{1}{(\sqrt{x}+\sqrt{y})^{2}} .
$$

Next we consider an integral

Noting that

$$
J(x)=\int_{b}^{\infty} K(x, y) \frac{d y}{y^{2}} .
$$

$$
(\sqrt{x}-\sqrt{y})^{2}>c \text { if and only if } y>(\sqrt{x}+c)^{2} \text { or } y<(\sqrt{x}-c)^{2},
$$

we have

$$
\begin{aligned}
J(x)= & \int_{(\sqrt{x}+\sqrt{c})^{2}}^{\infty}\left\{\frac{1}{(\sqrt{x}+\sqrt{y})^{2}}+\frac{1}{c}-\frac{1}{(\sqrt{x}-\sqrt{y})^{2}}\right\} \frac{d y}{y^{2}} \\
& +I(\sqrt{b}<\sqrt{x}-\sqrt{c}) \int_{b}^{(\sqrt{x}-\sqrt{c})^{2}}\left\{\frac{1}{(\sqrt{x}+\sqrt{y})^{2}}+\frac{1}{c}-\frac{1}{(\sqrt{x}-\sqrt{y})^{2}}\right\} \frac{d y}{y^{2}} \\
& +\int_{\max \left\{b,(\sqrt{x}-\sqrt{c})^{2}\right\}}^{(\sqrt{x}+\sqrt{c})^{2}} \frac{1}{(\sqrt{x}+\sqrt{y})^{2}} \frac{d y}{y^{2}} .
\end{aligned}
$$

Hence, if $\sqrt{x}>\sqrt{b}+\sqrt{c}$,

$$
J(x)=\int_{b}^{\infty} \frac{1}{(\sqrt{x}+\sqrt{y})^{2}} \frac{d y}{y^{2}}+\left(\int_{(\sqrt{x}+\sqrt{c})^{2}}^{\infty}+\int_{b}^{(\sqrt{x}-\sqrt{c})^{2}}\right)\left(\frac{1}{c}-\frac{1}{(\sqrt{x}-\sqrt{y})^{2}}\right) \frac{d y}{y^{2}},
$$

which we denote by $J_{1}(x)$.

On the other hand, if $\sqrt{x}<\sqrt{b}+\sqrt{c}$,

$$
J(x)=\int_{b}^{\infty} \frac{1}{(\sqrt{x}+\sqrt{y})^{2}} \frac{d y}{y^{2}}+\int_{(\sqrt{x}+\sqrt{c})^{2}}^{\infty}\left(\frac{1}{c}-\frac{1}{(\sqrt{x}-\sqrt{y})^{2}}\right) \frac{d y}{y^{2}},
$$

which we denote by $J_{2}(x)$.

Using $J_{1}(x)$ and $J_{2}(x)$, we can write

$$
I(a, b, c)=\int_{a}^{\infty} J(x) \frac{d x}{x^{2}}=\int_{(\sqrt{b}+\sqrt{c})^{2}}^{\infty} J_{1}(x) \frac{d x}{x^{2}}+\int_{a}^{(\sqrt{b}+\sqrt{c})^{2}} J_{2}(x) \frac{d x}{x^{2}} .
$$

Now, by an elementary calculation, we can evaluate $J_{1}(x)$ and $J_{2}(x)$ as follows:

$$
\begin{aligned}
J_{1}(x)= & \frac{1}{b c}-\frac{8}{\sqrt{b} x^{3 / 2}}-\frac{4}{\sqrt{c} x^{3 / 2}}+\frac{4 \sqrt{b}}{x^{3 / 2}(x-b)}+\frac{8 \sqrt{c}}{x^{3 / 2}(x-c)}-\frac{4}{\sqrt{c} \sqrt{x}(x-c)} \\
& +\frac{6}{x^{2}}(\log (\sqrt{x}+\sqrt{b})-\log (\sqrt{x}-\sqrt{b})) \\
& +\frac{6}{x^{2}}(\log (\sqrt{x}+\sqrt{c})-\log (\sqrt{x}-\sqrt{c}))
\end{aligned}
$$


and

$$
\begin{aligned}
J_{2}(x)= & \frac{1}{b x}-\frac{4}{\sqrt{b} x^{3 / 2}}-\frac{2}{\sqrt{c} x^{3 / 2}}-\frac{2}{x^{3 / 2}(\sqrt{x}+\sqrt{b})} \\
& -\frac{4}{x^{3 / 2}(\sqrt{x}+\sqrt{c})}+\frac{x-c}{c x(\sqrt{x}+\sqrt{c})^{2}} \\
& -\frac{6}{x^{2}}(\log \sqrt{b}+\log \sqrt{b})+\frac{6}{x^{2}}(\log (\sqrt{x}+\sqrt{b})+\log (\sqrt{x}+\sqrt{c}))
\end{aligned}
$$

Substituting these into (3.5) we can obtain (3.4).

At this point let us consider a random triangle in the Euclidean plane, not in the hyperbolic plane. Let $\mathrm{ABC}$ be a triangle whose three vertices are mutually independent and uniformly distributed on the unit circle centered at the origin of the Euclidean plane. In the below we will consider a certain expectation concerning a random triangle $\mathrm{ABC}$. For this purpose, without loss of generality, we may assume that the vertex $\mathrm{A}$ lies on the $x$-axis. We denote the angles which the line segments $\mathrm{OB}$ and OC make with the $x$-axis by $\phi$ and $\psi$ respectively. Furthermore we denote $X=\mathrm{BC}, Y=\mathrm{CA}$ and $Z=\mathrm{AB}$. Then we have

$$
X=\sqrt{2(1-\lambda)}, \quad Y=\sqrt{2(1-\mu)}, \quad Z=\sqrt{2(1-v)}
$$

where

$$
\lambda=\cos (\psi-\phi), \quad \mu=\cos \psi, \quad v=\cos \phi .
$$

Using $X, Y, Z$, we define

$$
\begin{aligned}
k(X, Y, Z)= & \left(\frac{Y^{2}}{X^{2}}+\frac{X^{2}}{Y^{2}}+\frac{Z^{2}}{Y^{2}}+\frac{Y^{2}}{Z^{2}}+\frac{X^{2}}{Z^{2}}+\frac{Z^{2}}{X^{2}}\right) \\
& -2\left(\frac{Y^{2} Z}{X^{3}}+\frac{Y Z^{2}}{X^{3}}+\frac{Z^{2} X}{Y^{3}}+\frac{Z X^{2}}{Y^{3}}+\frac{X^{2} Y}{Z^{3}}+\frac{X Y^{2}}{Z^{3}}\right) \\
& -\frac{2}{3}\left(\frac{Z^{2}}{X Y}+\frac{X^{2}}{Y Z}+\frac{Y^{2}}{Z X}\right)+2\left(\frac{Y^{2} Z^{2}}{X^{4}}+\frac{Z^{2} X^{2}}{Y^{4}}\right) \log \frac{X+Y}{Z} \\
& +2\left(\frac{Z^{2} X^{2}}{Y^{4}}+\frac{X^{2} Y^{2}}{Z^{4}}\right) \log \frac{Y+Z}{X} \\
& +2\left(\frac{X^{2} Y^{2}}{Z^{4}}+\frac{Y^{2} Z^{2}}{X^{4}}\right) \log \frac{Z+X}{Y} .
\end{aligned}
$$

Then we obtain the following theorem.

THEOREM 3. The limit probability $p_{c}$ is given by an expectation concerning a random triangle in the Euclidean plane,

$$
p_{c}=1-E(k(X, Y, Z)) .
$$


Proof. If we put

$$
a=\sqrt{\frac{1-\lambda}{(1-\mu)(1-v)}}, \quad b=\sqrt{\frac{1-\mu}{(1-v)(1-\lambda)}}, \quad c=\sqrt{\frac{1-v}{(1-\lambda)(1-\mu)}},
$$

the assumption stated in Lemma 5 is equivalent to the condition that

$$
X<Y+Z, \quad Y<Z+X, \quad Z<X+Y,
$$

which are obviously satisfied. Therefore Lemma 4 and Lemma 5 establish the theorem.

\section{Numerical evaluation of the probability $p_{c}$} integral

In order to find the value $p_{c}$ by (3.7) in Theorem 3 , it suffices to evaluate the

$$
J=\frac{1}{2 \pi^{2}} \iint_{T} f(\phi, \psi) d \phi d \psi
$$

where the domain of integration is

$$
T=\{(\phi, \psi): 0 \leq \phi \leq 2 \pi, \phi \leq \psi \leq 2 \pi\}
$$

and the integrand is $f(\phi, \psi)=k(x, y, z)$ with

$$
x=\sqrt{2(1-\cos (\psi-\phi))}, \quad y=\sqrt{2(1-\cos \psi)}, \quad z=\sqrt{2(1-\cos \phi)} .
$$

However, regretfully, it seems that we can not evaluate this integral analytically. Thus, in the below, we carry out an integration of (4.1) numerically.

For this purpose we first investigate properties of the function $f$. Denote the vertices of $T$ by

$$
\mathrm{V}_{1}=(0,0), \quad \mathrm{V}_{2}=(0,2 \pi), \quad \mathrm{V}_{3}=(2 \pi, \pi)
$$

Then we can see the following lemma by an elementary calculus.

LEMMA 6. (i) In the interior of $T$, $f$ is a $C^{\infty}$ function.

(ii) On $T, f$ is bounded: $0 \leq f \leq 1$.

(iii) When $(\phi, \psi)$ approaches to any point on the boundary of $T$ except $\mathrm{V}_{1}, \mathrm{~V}_{2}$ and $\mathrm{V}_{3}, f$ converges to 1 .

Thus, except neighbourhoods of $\mathrm{V}_{1}, \mathrm{~V}_{2}$ and $\mathrm{V}_{3}$, the function $f$ behaves mildly. However, to compute values of $f$, we need some care. If we compute values of $f$ using (3.6) near the boundary of $T$ (outside neighbourhoods of $\mathrm{V}_{1}, \mathrm{~V}_{2}$ and $\mathrm{V}_{3}$ ), we may have unreliable values because one of $x, y, z$ is small. To get reliable values, we use the fact that $k$ is a symmetric function of $(x, y, z)$ and is invariant under multiplication by any constant, i.e., $k(\sigma x, \sigma y, \sigma z)=k(x, y, z)$ for any $\sigma>0$. Thus, supposing that $x \geq y \geq z$, we introduce variables 


$$
s=y / x \text { and } t=z / y .
$$

Then $k(x, y, z)$, or equivalently $f(\phi, \psi)$, can be written as

$$
\begin{aligned}
\left(s^{2}+\right. & \left.\frac{1}{s^{2}}+t^{2}+\frac{1}{t^{2}}+s^{2} t^{2}+\frac{1}{s^{2} t^{2}}\right) \\
& -2\left(s^{3} t+s^{3} t^{2}+\frac{t^{2}}{s}+\frac{t}{s}+\frac{1}{s^{2} t^{3}}+\frac{1}{s t^{3}}\right) \\
& -\frac{2}{3}\left(s t^{2}+\frac{1}{s^{2} t}+\frac{s}{t}\right)+2\left(s^{4} t^{2}+\frac{t^{2}}{s^{2}}\right) \log \frac{1+s}{s t} \\
& +2\left(\frac{t^{2}}{s^{2}}+\frac{1}{s^{2} t^{4}}\right) \log (s(1+t))+2\left(s^{4} t^{2}+\frac{1}{s^{2} t^{4}}\right) \log \frac{1+s t}{s} .
\end{aligned}
$$

Now, since $x, y, z$ stand for lengths of three sides of a triangle, we must have $x<y+z$, from which follows $s(1+t)>1$. In particular $s>1 / 2$ and only $t$ may be small.

For small $t$, considering (4.2) as a function of $t$ and expanding it as

$$
\begin{aligned}
\frac{1+s^{4}}{2 s} & -\frac{8\left(1+s^{5}\right)}{5 s^{2}} t \\
+ & \left\{2\left(s^{4}+\frac{1}{s^{2}}\right) \log 1+s-4 s^{4} \log s\right. \\
& \left.\quad-\frac{1}{3 s^{2}}\left(1+6 s-3 s^{2}+2 s^{3}-3 s^{4}+6 s^{5}+s^{6}\right)\right\} t^{2} \\
- & 2\left(s^{4}+\frac{1}{s^{2}}\right) t^{2} \log t+\frac{2}{s^{2}} t^{2} \log (1+t)+2 s^{4} t^{2} \log (1+s t) \\
+ & \frac{2}{s^{2}} \sum_{n=7}^{\infty} \frac{(-1)^{n-1}}{n}\left(1+s^{n}\right) t^{n-4} .
\end{aligned}
$$

we will compute values of $f$ using this expansion.

Now we explain a procedure for integrating $J$. Choose a large integer $n$ and put $\delta=2 \pi / n$. We divide the domain of integration $T$ into small trianglular regions,

where

$$
T=\left(\bigcup_{l=1}^{n} \bigcup_{J=l}^{n} T_{i j}^{(1)}\right) \cup\left(\bigcup_{l=1}^{n-1} \bigcup_{J=l+1}^{n} T_{i j}^{(2)}\right)
$$

$$
\begin{aligned}
& T_{i j}^{(1)}=\{(\phi, \psi):(i-1) \delta \leq \phi \leq i \delta, \quad \phi+(j-i) \delta \leq \psi \leq j \delta\}, \\
& T_{i j}^{(2)}=\{(\phi, \psi):(i-1) \delta \leq \phi \leq i \delta, \quad(j-1) \delta \leq \psi \leq \phi+(j-i) \delta\} .
\end{aligned}
$$

Our idea for integrating $J$ is simply to approximate the function $f$ by a linear 
function in each triangular region $T_{i j}^{(1)}$ and $T_{i j}^{(2)}$, except three triangular regions containing $\mathrm{V}_{1}, \mathrm{~V}_{2}$ and $\mathrm{V}_{3}$. To state the procedure in detail, we put

$$
\begin{aligned}
& f_{i j}=f(i \delta, j \delta) \text {, } \\
& \bar{f}_{i j}^{(1)}=\max \left\{f_{i j}, f_{l-1, J}, f_{l-1, J-1}\right\}, \quad \underline{f_{i j}}{ }^{(1)}=\min \left\{f_{i j}, f_{l-1, J}, f_{l-1, J-1}\right\} \text {, } \\
& \bar{f}_{i j}^{(2)}=\max \left\{f_{i j}, f_{l, J-1}, f_{l-1, J-1}\right\}, \quad \underline{f_{i j}^{(2)}}=\min \left\{f_{i j}, f_{l, J-1}, f_{l-1, J-1}\right\}, \\
& \tilde{f}_{i j}^{(1)}=\frac{1}{3}\left(f_{i j}+f_{l-1, J}+f_{l-1, J-1}\right), \quad \tilde{f}_{i j}^{(2)}=\frac{1}{3}\left(f_{i j}+f_{l, J-1}+f_{l-1, J-1}\right) .
\end{aligned}
$$

Then we compute

$$
\begin{gathered}
\overline{J_{n}}=\frac{\delta^{2}}{2}\left(\sum^{(1)}{\overline{f_{i j}}}^{(1)}+\sum^{(2)}{\overline{f_{i j}}}^{(2)}+3\right), \\
\underline{J_{n}}=\frac{\delta^{2}}{2}\left(\sum^{(1)}{\underline{f_{i j}}}^{(1)}+\sum^{(2)}{\underline{f_{i j}}}^{(2)}\right), \\
\widetilde{J}_{n}=\frac{\delta^{2}}{2}\left(\sum^{(1)}{\widetilde{f_{i j}}}^{(1)}+\sum^{(2)}{\tilde{f_{i j}}}^{(2)}\right),
\end{gathered}
$$

where the summation $\sum^{(1)}$ is taken over

$$
\{(i, j): i=1 \text { and } 2 \leq j \leq n-1\} \cup\{(i, j): 1 \leq i \leq n-1, i \leq j \leq n\}
$$

and $\sum^{(2)}$ taken over

$$
\{(i, j): 1 \leq i \leq n-1, i+1 \leq j \leq n\} .
$$

Note that the addition by 3 in $\overline{J_{n}}$ is due to overestimating $f$ in the three triangular regions containing $\mathrm{V}_{1}, \mathrm{~V}_{2}$ and $\mathrm{V}_{3}$. Since $f$ behaves mildly outside neighbourhoods of $\mathrm{V}_{1}, \mathrm{~V}_{2}$ and $\mathrm{V}_{3}$, we may reasonably expect that $J_{n}<J<\overline{J_{n}}$ for large $n$ and $\widetilde{J}_{n}$ is nearly equal to $J$.

Now we give results of numerical integration in Table 1 below.

TABLE 1. Results of numerical integration.

\begin{tabular}{cccc}
\hline$n$ & $\underline{J_{n}}$ & $\overline{J_{n}}$ & $\widetilde{J}_{n}$ \\
\hline 10000 & 0.4594403380 & 0.4597998565 & 0.4596201032 \\
20000 & 0.4595303987 & 0.4597101697 & 0.4596202857 \\
30000 & 0.4595603966 & 0.4596802464 & 0.4596203221 \\
40000 & 0.4595753909 & 0.4596652793 & 0.4596203355 \\
50000 & 0.4595843860 & 0.4596562972 & 0.4596203418 \\
60000 & 0.4595903821 & 0.4596503083 & 0.4596203454 \\
70000 & 0.4595946646 & 0.4596460302 & 0.4596203475 \\
80000 & 0.4595978764 & 0.4596428213 & 0.4596203490 \\
90000 & 0.4596003743 & 0.4596403254 & 0.4596203499 \\
100000 & 0.4596023726 & 0.4596383286 & 0.4596203507 \\
\hline
\end{tabular}


TABLE 2. Estimates and standard errors for parameters.

\begin{tabular}{ccc}
\hline parameters & estimates & standard errors \\
\hline$\underline{p_{c}}$ & 0.4596203893 & $7.23269 \times 10^{-9}$ \\
$\overline{p_{c}}$ & 0.4596203967 & $8.85162 \times 10^{-9}$ \\
$\widetilde{p_{c}}$ & 0.4596203921 & $7.83131 \times 10^{-9}$ \\
$\underline{m}$ & -1.800335448 & 0.000183724 \\
$\bar{m}$ & +1.794814854 & 0.000224848 \\
$\tilde{m}$ & -0.0026965198 & 0.000198930 \\
\hline
\end{tabular}

To these data we fit linear functions of $1 / n$,

$$
\underline{J_{n}}=\underline{p_{c}}+\underline{m} \cdot \frac{1}{n}, \quad \overline{J_{n}}=\overline{p_{c}}+\bar{m} \cdot \frac{1}{n}, \quad \widetilde{J}_{n}=\widetilde{p}_{c}+\tilde{m} \cdot \frac{1}{n},
$$

in the simple least square sence. Estimates and standard errors for parameters in the above linear functions are given in Table 2. From these results, we conclude that $p_{c}$ is nearly equal to 0.45962039 .

\title{
REFERENCES
}

[1] V. S. Alagar, On the distribution of a random triangle, J. Appl. Probab., 14 (1977), 284-297.

[2] C. Buchta, A note on the volume of a random polytope in a tetrahedron, Illinois J. Math., 30 (1986), 653-659.

[ 3 ] B. Eisenberg AND R. Sullivan, Random triangles in $n$ dimensions, Amer. Math. Monthly, 103 (1996), 308-318.

[4] W Fenchel, Elementary Geometry in Hyperbolic Space, Walter de Gruyter, Berlin, 1989.

[5] R. K. GuY, There are three times as many obtuse-angled triangles as there are acute-angled ones, Math. Mag., 66 (1993), 175-178.

[6] G. R. Hall, Acute triangles in the $n$-ball, J. Appl. Probab., 19 (1982), 712-715.

[7] D. G. Kendall AND H. L. LeE, The structure and explicit determination of convex-polygonally generated shape-densities, Adv. in Appl. Probab., 19 (1987), 896-916.

[ 8 ] W. S. Kendall, Symbolic computation and the diffusion of shapes of triads, Adv. in Appl. Probab., 20 (1988), 775-797.

[9] D. MANNiON, Convergence to collinearity of a sequence of random triangle shapes, Adv. in Appl. Probab., 22 (1990), 831-844.

[10] S. Portnoy, A Lewis Carrol pillow problem: probability of an obtuse triangle, Statıst. Sc1., 9 (1994), 279-284.

[11] W S. Woolhouse, Solution by the proposer to problem 1350, Mathematical Questions, with their Solutions from the Educational Times, C. F Hodgson and Son, London, 1886.

\author{
Department of Mathematics Education \\ KAGOSHIMA UNIVERSITY \\ KAGOSHIMA 890-0065 \\ JAPAN \\ e-mail: 1sokawa@rike1.edu.kagoshıma-u.ac.jp
}

